\title{
Transition from unstable electrostatic confinement to stable magnetic confinement in a helicon reactor operating with $\mathrm{Ar} \mathrm{SF}_{6}$ gas mixtures
}

\author{
C. S. Corr, ${ }^{\text {a) }}$ N. Plihon, and P. Chabert \\ Laboratoire de Physique et Technologie des Plasmas, Ecole Polytechnique, \\ 91128 Palaiseau Cedex, France
}

(Received 27 September 2005; accepted 5 March 2006; published online 25 May 2006)

\begin{abstract}
Two types of instabilities were previously identified in inductive discharges having an expanding chamber when negative ions are present: (i) the source instability, occurring in the neighborhood of the capacitive-to-inductive ( $E$ to $H$ ) transition, and (ii) the downstream instability, which was shown to be the periodic formation and propagation of double layers. These unstable double layers were found over the entire parameter space (pressure/power) of interest, and they were born at the interface of the source and diffusion chambers. They acted as an internal electrostatic barrier separating a low-electronegativity, high-electron-density plasma upstream (in the source) and a high-electronegativity, low-electron-density plasma downstream. In this paper we have investigated the effect of adding a static axial magnetic field, classically used to increase the confinement and the plasma heating via helicon wave propagation. This had the following consequences: (i) the unstable double layers, and therefore the axial electrostatic confinement, were suppressed in a large part of the parameter space, and (ii) the magnetic confinement leads to a radially stratified plasma, the center being a low-electronegativity, high-density plasma and the edges being essentially an ion-ion plasma. (C) 2006 American Institute of Physics. [DOI: 10.1063/1.2191431]
\end{abstract}

\section{INTRODUCTION}

High-density plasma sources operating with electronegative gases are widely used for deep etching in the micronanotechnologies. One system capable of delivering the high plasma densities required is the helicon plasma source. ${ }^{1} \mathrm{Heli}-$ con (or whistler) waves are a simplified form of right hand polarized electromagnetic waves in a magnetized plasma with frequencies between the lower-hybrid and electron cyclotron frequencies. Generally the plasma is ignited in a source chamber by an antenna operating at radio frequencies and allowed to diffuse into a chamber of larger dimensions.

Previously, while operating with mixtures of argon and $\mathrm{SF}_{6}$ in an expanding plasma, we reported instabilities due to the periodic formation and propagation of double layers born at the interface between the source and the diffusion chambers while operating without the static magnetic field ${ }^{2}$ (these double layers were stable in a very narrow range of electronegativity $\left.{ }^{3}\right)$. Relaxation oscillations between the capacitive and inductive modes, referred as the $E / H$ source instability, ${ }^{4-13}$ were also observed. With both types of instability, periodic modulations in the light output, floating potential, negative ion fraction, electron, and positive and negative ion densities were observed and measured.

Under certain conditions of electronegativity, input power and gas pressure, and while operating without the magnetic field, we were able to obtain stationary double layers which resulted in the separation of the plasma into a

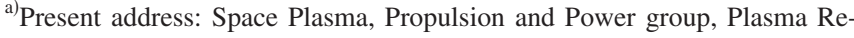
search Laboratory, RSPhysSE, The Australian National University, Canberra, ACT 0200, Australia; electronic mail: cormac.corr@anu.edu.au
}

high-density, low-electronegative plasma in the source and a low-density, high-electronegative plasma in the diffusion chamber. The main goal of this paper is to investigate the effect that an applied static magnetic field has on the plasma structure and stability. With the application of a magnetic field, which helps to produce high degrees of ionization, the motion of charged particles is profoundly modified when the Larmor radius is small compared to the system size. Under the magnetic field magnitudes explored in this paper, the electrons were magnetized while the ions were not. The presence of negative ions further complicates matters as they change the mass, and therefore the Larmor radii, of negatively charged particles. Indeed, it has previously been observed $^{14,15}$ and modeled ${ }^{16}$ that electronegative discharges with an applied static magnetic field can produce a region, at the plasma periphery, which is preferentially an ion-ion plasma. This type of structure could be very beneficial in plasma processing where wafer charging could be greatly reduced $^{17}$ and could serve as an alternative to pulse modulated discharges of electronegative gases. ${ }^{18}$

A further consequence of applying a static magnetic field is the propagation of the helicon wave, which becomes a significant heating mechanism at high power. Hence, a transition from inductive $(H)$ to helicon $(W)$ modes occurs. From previous work on the $E / H$ source instability, one would expect an $H / W$ source instability to develop. This will also be discussed in the paper.

The paper is organized as follows. In Sec. II the experimental setup is described. Section III presents the results and discussion on instabilities while Sec. IV focuses on the radial plasma structure due to the magnetic field. In Sec. V some conclusions will be drawn. 


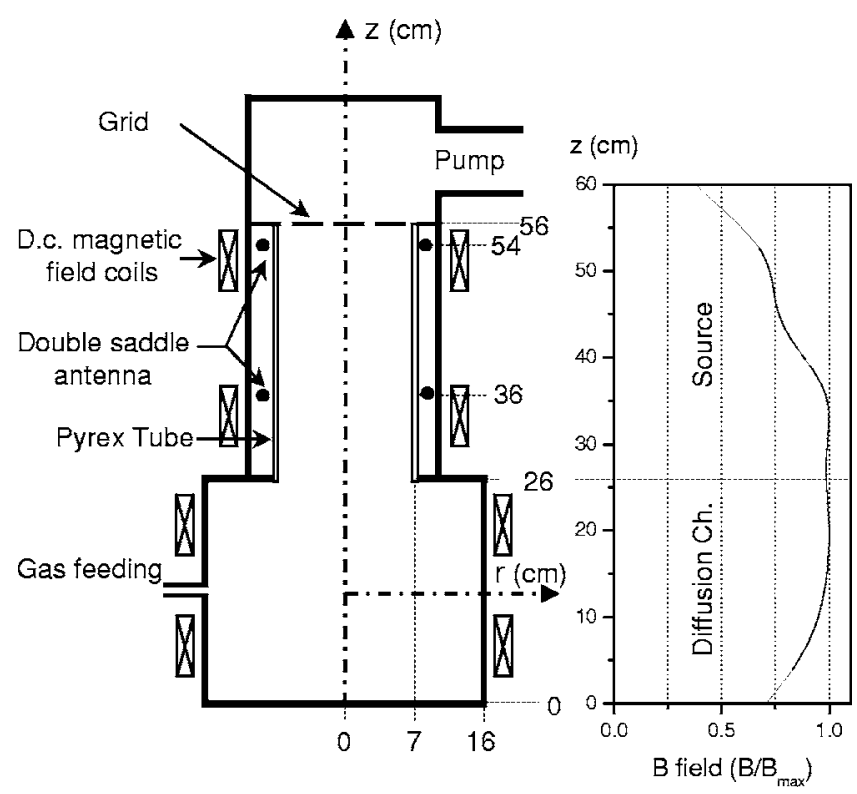

FIG. 1. A schematic of the helicon chamber and the calculated axial magnetic field amplitude along the symmetry axis of the reactor.

\section{EXPERIMENTAL ARRANGEMENT AND DIAGNOSTICS}

A schematic diagram of the helicon plasma source and the calculated axial magnetic field strength along the reactor axis used in all experiments are shown in Fig. 1. The reactor consists of a source and a diffusion chamber [Fig. 1(a)]. The source is a $14 \mathrm{~cm}$ diameter, $30 \mathrm{~cm}$ long and $0.9 \mathrm{~cm}$ thick Pyrex cylinder surrounded by a double saddle field-type helicon antenna. ${ }^{19}$ The fan-cooled antenna is powered through a close-coupled $L$-type matching network by a rf power supply operating at $13.56 \mathrm{MHz}$ and capable of delivering $2 \mathrm{~kW}$ forward power. The input power was recorded as the difference between the forward and reflected powers. The Pyrex cylinder is housed in an aluminum cylinder of $20 \mathrm{~cm}$ diameter and $30 \mathrm{~cm}$ long surrounded by two magnetic field coils and sits atop a $32 \mathrm{~cm}$ diameter, $24 \mathrm{~cm}$ long diffusion chamber also surrounded by two field coils. The currents in the coils were such that the most uniform magnetic field lines for this configuration were obtained [Fig. 1(b)]. A plasma column can be observed in the diffusion chamber directly under the source region. When referring to the magnetic field amplitude, we refer to the maximum amplitude along the axis (namely, at the interface between the source and diffusiion chamber, at $z=26 \mathrm{~cm}$ and $r=0 \mathrm{~cm}$ ). The maximum magnitude used in this paper is $34 \mathrm{G}$. A turbomolecular pump attached to the other end of the source tube routinely achieves base pressures of $10^{-6}$ Torr before gas $\left(\mathrm{Ar}\right.$ and $\left.\mathrm{SF}_{6}\right)$ is flowed through the diffusion chamber and the flow rate adjusted to obtain the desired operating pressure.

The optical emission from the plasma was collected using a fast photodiode and the floating potential measured using a Langmuir probe to detect unstable behavior over the frequency range from $1 \mathrm{~Hz}$ to $500 \mathrm{kHz}$. A passively compensated Langmuir probe, ${ }^{20}$ of $0.25 \mathrm{~mm}$ diameter and $5 \mathrm{~mm}$ long platinum wire tip, was used to determine the plasma parameters from measurements of the probe $I(V)$ character- istics using a SMARTSOFT data acquisition system. ${ }^{21}$ The positive ion saturation current was measured using a nickel planar probe with guard ring biased at the same potential as the probe. The diameter of the collecting area was $4 \mathrm{~mm}$ and the diameter of the outer ring was $8 \mathrm{~mm}$. The negative ion fraction, $\alpha=n_{-} / n_{e}$, could therefore be determined from the ratio of the ion and the electron saturation currents using the double probe technique described in Chabert et al. ${ }^{15}$ using the theory of Sheridan et al $^{22}$ The ratio of the electron temperature to the negative ion temperature, $\gamma=T_{e} / T_{-}$, was taken to be 15 as is commonly thought. ${ }^{15,22}$ The mass of the positive ions was calculated from a standard average for the mixtures where the dominant ions were taken to be $\mathrm{SF}_{3}^{+}$ and $\mathrm{Ar}$ as has been previously observed at these low pressures. ${ }^{23,24}$ The positive ion density was calculated assuming charge neutrality $n_{+}=n_{-}+n_{e}$. All radial measurements were carried out in the diffusion chamber, $12 \mathrm{~cm}$ from the source, and in each figure the edge of the source chamber is represented by the line.

\section{UNSTABLE AXIAL ELECTROSTATIC CONFINEMENT}

Previously, we reported experimental results concerning two types of instability that are observed in our helicon reactor when operating without the magnetic field, i.e., only the capacitive and inductive modes were achievable. These were relaxation oscillations, related to capacitive-toinductive mode transitions, and a downstream instability. ${ }^{2}$ The relaxation oscillation amplitudes were observed to be strongest in the source chamber, decayed on moving downstream into the diffusion chamber while remaining in phase. They occurred for relatively low input powers (100$200 \mathrm{~W}$ ). At higher input power a downstream instability became apparent with a smaller amplitude modulation compared with that of the source instability. This instability displayed a maximum close to the source/diffusion chamber interface, moved out of phase on traveling downstream into the diffusion chamber and was shown to be related to the periodic formation and propagation of a double layer occurring only for a certain fraction of negative ions. ${ }^{2}$ In fact, there is a small overlap in the input power where both instabilities can simultaneously occur.

When operating with a static magnetic field the two types of instability were observed with similar behavior to that described above. However, the total power and pressure window of instability was reduced. The instability window of power and pressure is shown in Fig. 2 for (a) the no magnetic field case, (b) a magnetic field of $17 \mathrm{G}$, and (c) a magnetic field of $34 \mathrm{G}$. When the discharge was operated with no magnetic field the plasma was essentially unstable everywhere, with a relatively small window for the source instability, and a very large window for the downstream instability. Application of the magnetic field causes an increase in the source instability window but reduces the downstream instability window. The window reduces with increasing the magnetic field magnitude, suggesting that the downstream instability can be suppressed with a very high field. The effect is greater at low pressures ( $<5$ mTorr) where the mag- 

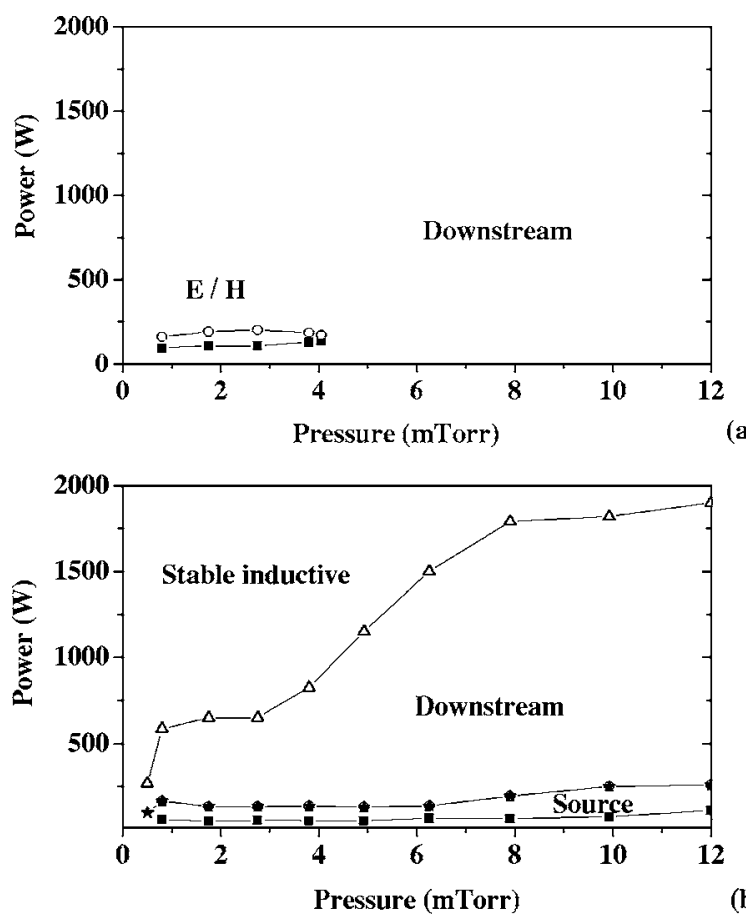

(b)

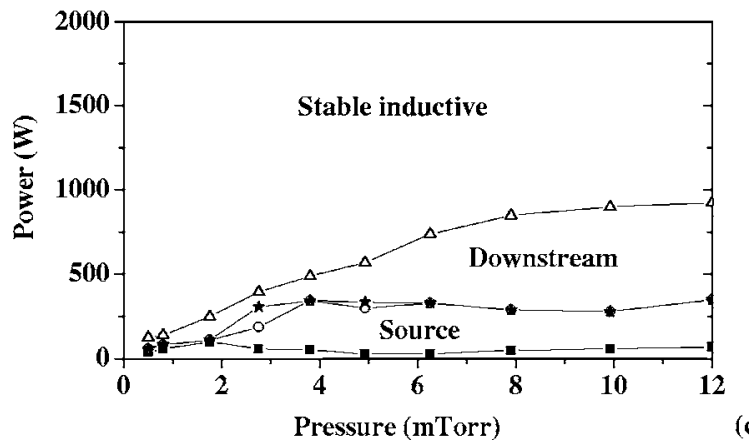

(c)

FIG. 2. The instabily windows for a 1:1 Ar/ $\mathrm{SF}_{6}$ discharge at $1 \mathrm{mTorr}$ and $500 \mathrm{~W}$ for (a) no magnetic field, (b) a magnetic field of $17 \mathrm{G}$, and (c) a magnetic field of $34 \mathrm{G}$.

netic confinement is efficient. At higher pressure, the magnetic confinement is greatly reduced returning to the no-field case diffusion conditions, thus allowing for double layers to form.

The reason why the source instability window increases when increasing the magnetic field magnitude remains unclear. The inductive power absorption dependence on the electron density may be modified in such a way that the relaxation oscillation window is increased. Indeed, Chabert et $a .^{5}$ have previously shown that the position of the inductive peak power curve and thus the inductive-to-capacitive power ratio is a key parameter in determining the window of existence of the relaxation oscillations near the $E$-to- $H$ transition.

We explored the change in the time-averaged axial plasma parameters on going from an unstable discharge to a stable discharge by increasing the input power. Figure 3 shows the axial dependence of the electron density for operating powers of $200 \mathrm{~W}$ (unstable) and $800 \mathrm{~W}$ (stable) at 1 mTorr and 17 G. For $200 \mathrm{~W}$ a steep gradient is observed between 22 and $24 \mathrm{~cm}$ which is related to the formation of a propagating double layer. The steepest density gradient is

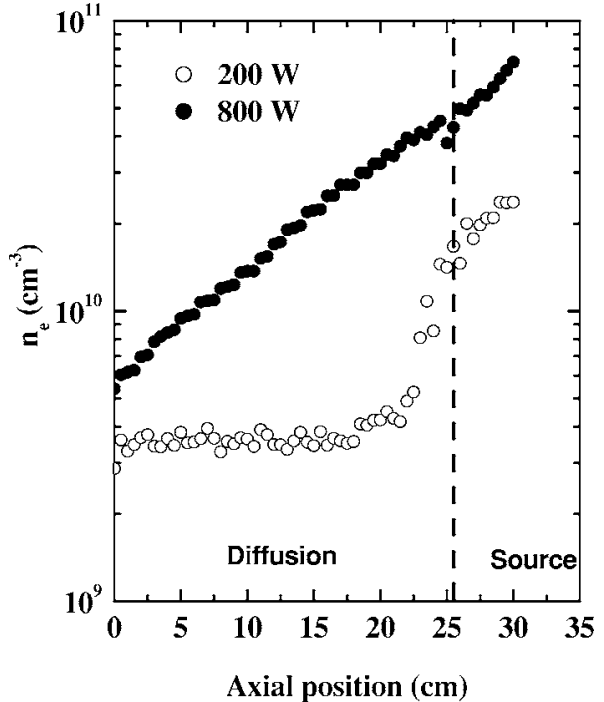

FIG. 3. The axial variation of the electron density measured at $1 \mathrm{mTorr}$ and $17 \mathrm{G}$.

observed at $23 \mathrm{~cm}$ which is the averaged position of the moving double layer. A similar trend is observed in the plasma potential which decreases by $7 \mathrm{~V}$ over $2 \mathrm{~cm}$ (Fig. 4). This is similar to our previous observations without the magnetic field. ${ }^{2}$ Increasing the input power results in a quiescent plasma state, as already shown on the instability windows, and in a smooth gradient of the electron density which gradually decays from the source to the bottom of the diffusion chamber. The unstable double layers were thus suppressed and a bright plasma column was observed underneath the source.

Another phenomenon is observed when operating with the magnetic field. At low pressures ( $<5$ mTorr) and over a small range of input powers the discharge passes from the downstream instability to a quiescent discharge and then to an unstable discharge once more. This third instability may be a source instability related to the transition from the in-

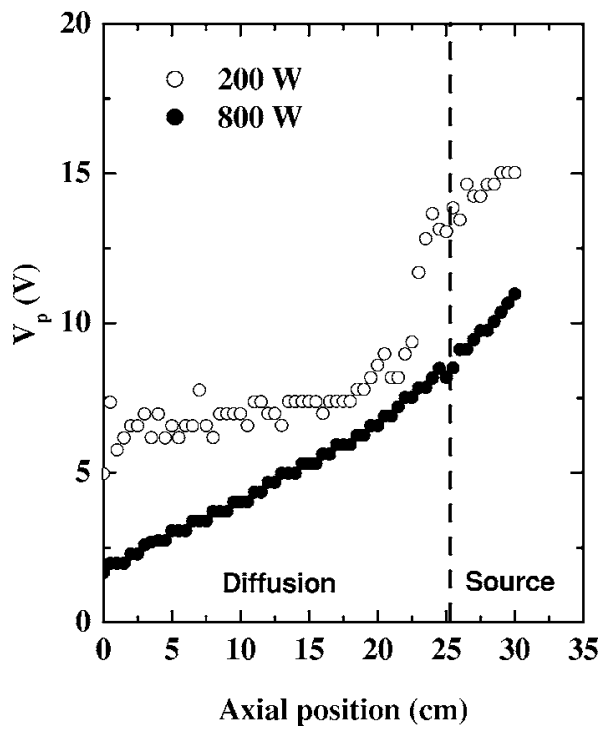

FIG. 4. The axial variation of the plasma potential measured at $1 \mathrm{mTorr}$ and $17 \mathrm{G}$. 


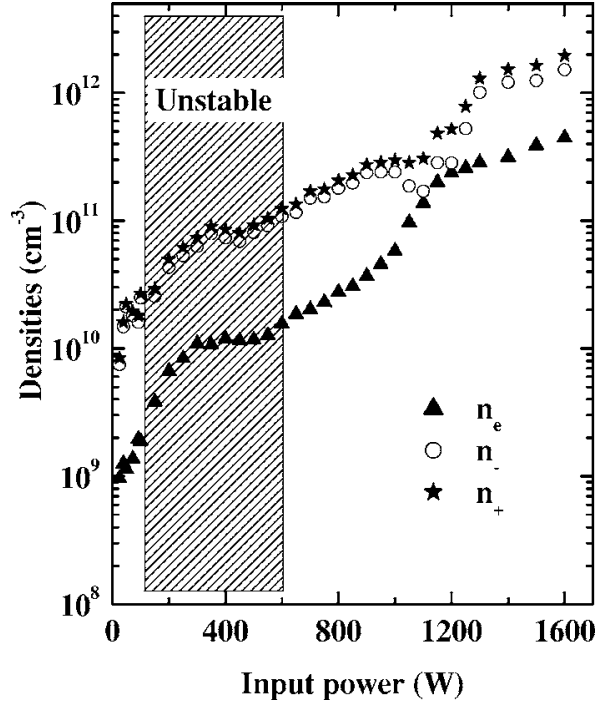

FIG. 5. The electron and ion density dependences on power for a 1:1 $\mathrm{Ar} / \mathrm{SF}_{6}$ discharge with the magnetic field at $1 \mathrm{mT}$ orr and $17 \mathrm{G}$.

ductive mode to the helicon wave mode. To further explore our observations we measured the charged particle densities as a function of input power. These results are presented in Fig. 5 for an applied magnetic field of $17 \mathrm{G}$ and a gas pressure of $1 \mathrm{~m}$ Torr. Generally density jumps are expected with increasing power, that would correspond, in our case, to transitions from the capacitive mode to the inductive mode and then to the helicon wave mode. Here we do not experience density jumps. However, all densities are observed to increase with increasing input power although not linearly which suggest transitions to the different operating modes. The large $E$-to- $H$ instability region for these particular conditions of pressure and magnetic field is shown by the shaded area. The $H$-to- $W$ oscillations mentioned above occurred at around $1200 \mathrm{~W}$ (for a very narrow range of powers), where we observe a sharp change in the densities.

An interesting and surprising point to note is the increase in the negative ion fraction with increasing power above $1200 \mathrm{~W}$. Generally, the negative ion fraction is observed to decrease with increasing power ${ }^{25}$ due to an increase in negative ion loss processes such as detachment by electron impact (small cross section), detachment through association with a $\mathrm{SF}_{x}$ radical (e.g., $\mathrm{F}^{-}+\mathrm{SF}_{x} \rightarrow \mathrm{SF}_{x+1}+e^{-}$), the density of radicals being larger at higher powers, and recombination with positive ions in the volume.

\section{TRANSITION TO RADIAL MAGNETIC CONFINEMENT}

Although the application of a magnetic field suppresses the axial plasma confinement, it does, however, lead to a radial confinement. The radial profile of the negative ion fraction is shown in Fig. 6 for various values of the magnetic field. With no field the negative ion fraction is $\approx 30$ across the radius and decreases near the chamber wall. In this case, the downstream plasma is highly electronegative since it is separated from the source by the double layer. If the magnetic field is now switched on and increased gradually, the negative ion fraction on the center axis decreases while it

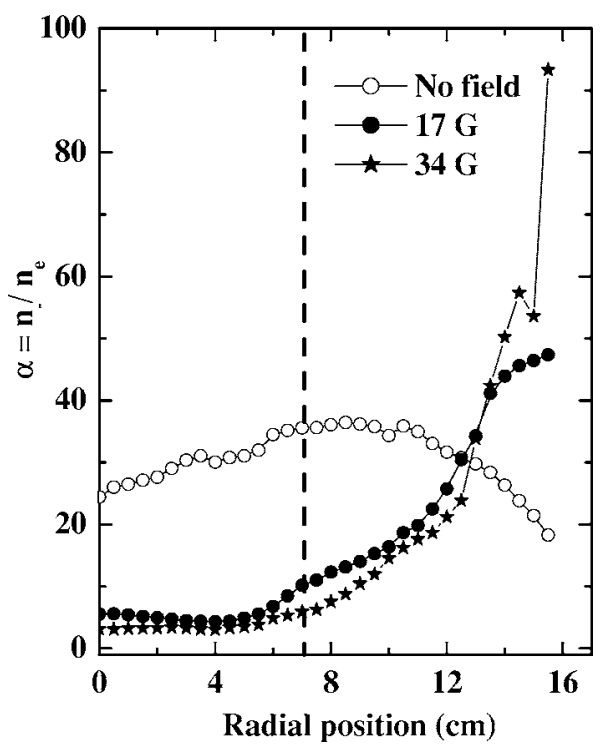

FIG. 6. The radial negative ion fraction dependence on the magnetic field at $1 \mathrm{~m}$ Torr and $600 \mathrm{~W}$.

increases in the edge region. This occurs because the double layer is no longer present and the low-electronegativity plasma column (of radius $4-6 \mathrm{~cm}$ ) fills the diffusion chamber. Although the negative ion fraction is larger towards the edge of the diffusion chamber, this does not mean that the negative ion density is greater compared to the center. In fact, for these conditions, the positive ion and negative ion densities remain mostly constant over the radius. However, the radial distribution of the electrons is greatly modified. To quantify the radial stratification of the electronegativity $\alpha$, we plot on Fig. 7 the ratio of $\alpha$ in the center to the edge as a function of the magnetic field magnitude. This ratio almost reaches zero above a few tens of gauss showing that an ionion plasma is formed at the periphery of the diffusion chamber.

In comparison with the electron density, the ion density profiles are not profoundly modified by the magnetic field

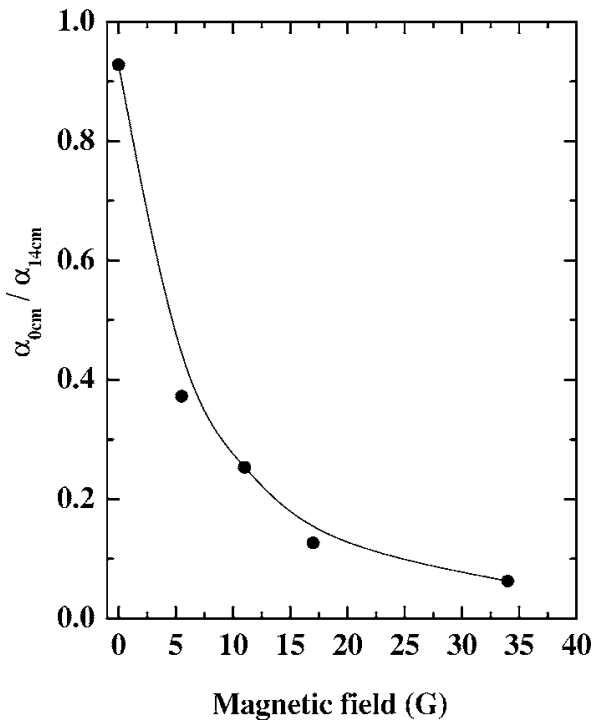

FIG. 7. The negative ion fraction center to edge $(14 \mathrm{~cm})$ ratio vs magnetic field. 

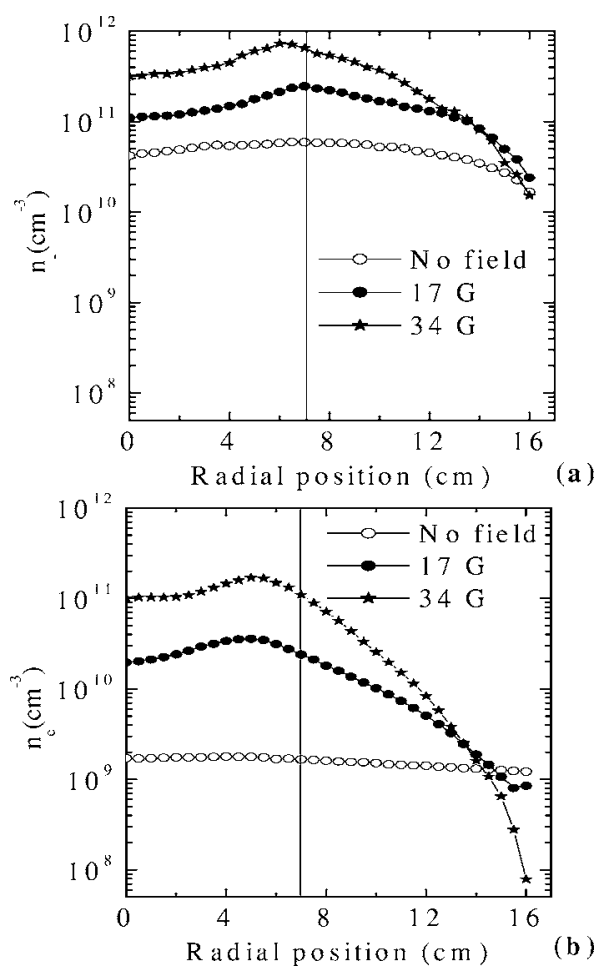

FIG. 8. The radial dependence of the (a) negative ion density and (b) the electron density on the magnetic field at 1 mTorr and $600 \mathrm{~W}$.

(Fig. 8). The ion densities decrease slightly from the center to the edge (by approximately a factor of 3 for $17 \mathrm{G}$ and 10 for $34 \mathrm{G}$ ), although they may exhibit a small dip in the central region which may be due to an increase in ion-ion recombination due to a larger number of positive ions flowing from the source chamber. This is shown in Fig. 8(a). Figure 8(b) shows the radial profile of the electron density for various magnetic field magnitudes. For no field, the electron density decays slowly from the center to the edge, with a central density of $2 \times 10^{9} \mathrm{~cm}^{-3}$. At $17 \mathrm{G}$, the profile exhibits a sharp gradient for radial positions greater than $7 \mathrm{~cm}$ (which corresponds to the edge of the source chamber). The central density increases by a factor of 10 while the density remains about $10^{9} \mathrm{~cm}^{-3}$ at the wall of the diffusion chamber. The maximum of the electron density is located at $r \approx 6 \mathrm{~cm}$, that is at the edge of the high-density plasma column. This indicates a maximum of power deposition here, which will be confirmed by electron temperature measurements as we shall see below. This electron heating and confinement feature increases when increasing the magnetic field (compare 17 and $34 \mathrm{G}$ cases).

The confinement behavior observed is because the electrons experience the effect of the field, having a Larmor radius of a few millimeters while that of the ions is greater than the discharge radius. Diffusion for electrons is now preferentially along the axis whereas for ions it remains isotropic. The discharge is now separated into two regions: an electron-ion plasma column in the center under the source region and an ion-ion plasma near the walls. This has previously been modeled by Franklin and Snell ${ }^{16}$ and has been observed experimentally in $\mathrm{CF}_{4}$ by Kawai and Mieno. ${ }^{14} \mathrm{~A}$ further indication of an inner electron-ion plasma column

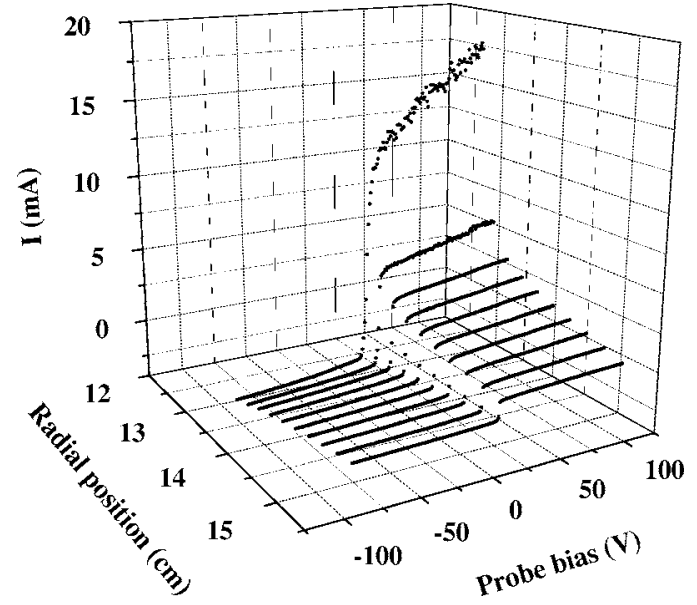

FIG. 9. The radial dependence of the $I(V)$ chatacteristics measured by the planar probe at $1 \mathrm{mT}$ Torr and $600 \mathrm{~W}$ and a magnetic field of $34 \mathrm{G}$.

and an outer ion-ion plasma being formed is from the $I(V)$ characteristics obtained by the planar probe, as shown in Fig. 9. At the plasma edge the negative ions are the dominant source of the negative particle current, the positive and negative currents being nearly equal, giving the symmetric probe characteristic. When approaching the plasma column, the electron current quickly dominates leading to a much larger negative particle current than the positive particle current.

The strong electronegativity at the plasma edge can also be explained by the radial profile of the electron temperature which is shown in Fig. 10 for various magnetic field magnitudes. The operating conditions are the same as for Fig. 8. For the no field 1:1 Ar/SF 6 discharge, the electron temperature is relatively flat across the radius with values of $\approx 3.4 \mathrm{eV}$ at the center and $\approx 3 \mathrm{eV}$ at the edge, therefore, giving a large and uniform electron attachment coefficient across the radius. With the application of the magnetic field the radial profile is now drastically changed with a maximum of $\approx 4.4 \mathrm{eV}$ occurring at $r \approx 6 \mathrm{~cm}$ for the $34 \mathrm{G}$ case. In the

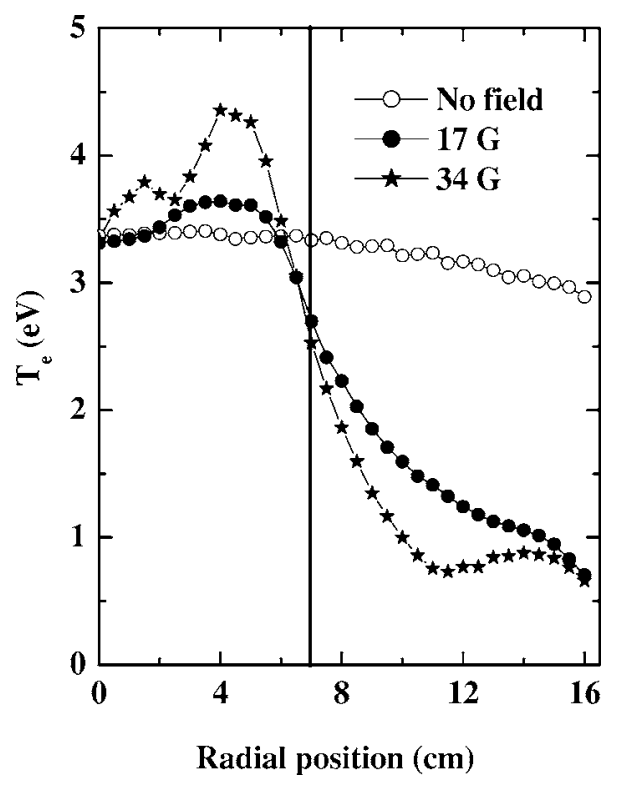

FIG. 10. The radial electron temperature dependence on the magnetic field at $1 \mathrm{mTorr}$ and $600 \mathrm{~W}$. 


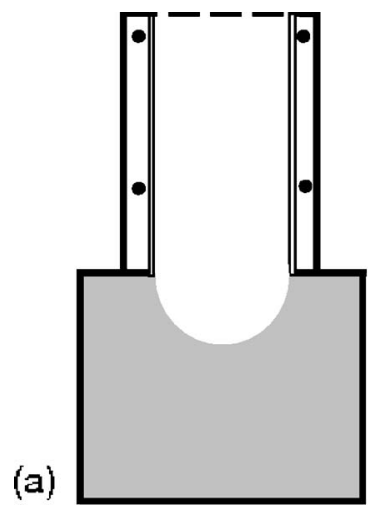

(b)

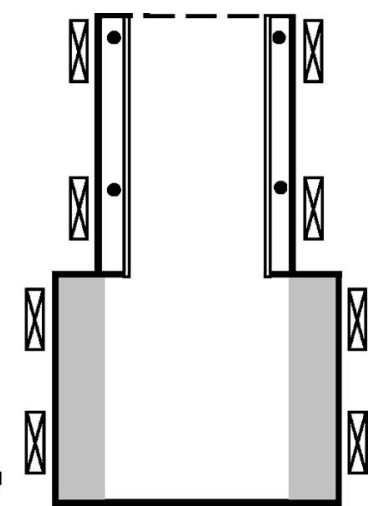

FIG. 11. (a) Axial electrostatic and (b) radial magnetic confinements. The shaded region corresponds to highly electronegative plasmas.

center the electron temperature is $3.5 \mathrm{eV}$ and towards the diffusion chamber walls this falls as low as $0.6 \mathrm{eV}$. These hot electrons seem to be located at the edge of the plasma column located in the diffusion chamber directly below the source chamber. This may be due to the fact that at $600 \mathrm{~W}$ the plasma is in the inductive mode (see Fig. 5), resulting in a large number of electrons being heated in the skin depth near the helicon antenna, that is at $r \approx 4-6 \mathrm{~cm}$. These hot electrons are efficiently transported along the axial magnetic field lines ( $z$ axis) to produce a tube (rather than a cylinder) of efficient ionization. The low temperature region near the diffusion chamber walls will favor electron attachment processes such as $\mathrm{SF}_{6}+e^{-} \rightarrow \mathrm{SF}_{6}^{-}, \mathrm{SF}_{6}+e^{-} \rightarrow \mathrm{SF}_{5}^{-}+\mathrm{F}$, and $\mathrm{SF}_{6}$ $+e^{-} \rightarrow \mathrm{F}^{-}+\mathrm{SF}_{5}$, and may therefore be an efficient production region for negative ions. ${ }^{26}$

\section{CONCLUSION}

We have investigated the effect of adding a magnetic field on the structure and the stability of a low pressure electronegative inductive/helicon discharge. We showed that the overall stability of the plasma was significantly improved. The downstream instability, which is due to the formation and propagation of double layers and represents the major cause of instability, can be almost suppressed. The source instability window (relaxation oscillations in the neighborhood of the $E$-to- $H$ transition) was slightly increased but remain much smaller than the usual parameter space used for plasma processing.

Our main conclusion for this work is that by applying a magnetic field, the plasma experiences a transition from un- stable electrostatic confinement to stable radial magnetic confinement. The first is due to double-layer formation at the source and diffusion chamber interface, whereas the second is due to the difference in Larmor radii between electrons and both positive and negative ions. Figure 11 shows the two types of confinement described above. The first drawing shows the axial stratification due to the formation of double layers, while the second shows the radial magnetic confinement.

${ }^{1}$ R. W. Boswell, Plasma Phys. Controlled Fusion 26, 1147 (1984).

${ }^{2}$ N. Plihon, C. S. Corr, and P. Chabert, J. Appl. Phys. 98, 023306 (2005).

${ }^{3}$ N. Plihon, C. S. Corr, and P. Chabert, Appl. Phys. Lett. 86, 091501 (2004).

${ }^{4}$ M. A. Lieberman, A. J. Lichtenberg, and A. M. Marakhtanov, Appl. Phys. Lett. 75, 3617 (1999).

${ }^{5}$ P. Chabert, A. J. Lichtenberg, M. A. Lieberman, and A. M. Marakhtanov, Plasma Sources Sci. Technol. 10, 478 (2001).

${ }^{6}$ A. J. Lichtenberg, P. Chabert, M. A. Lieberman, and A. M. Markhtanov, Bifurcation Phenomena in Plasmas (2001), p. 3.

${ }^{7}$ P. Chabert, H. Abada, J. P. Booth, and M. A. Lieberman, J. Appl. Phys. 94, 76 (2003).

${ }^{8}$ P. Chabert, A. J. Lichtenberg, M. Lieberman, and A. M. Marakhtanov, J. Appl. Phys. 94, 831 (2003).

${ }^{9}$ M. Tuszewski and R. R. White, J. Appl. Phys. 94, 2858 (2003).

${ }^{10}$ M. Tuszewski, R. R. White, and G. A. Wurden, Plasma Sources Sci. Technol. 12, 396 (2003).

${ }^{11}$ A. M. Marakhtanov, M. Tuszewski, M. A. L. A. J. Lichtenberg, and P. Chabert, J. Vac. Sci. Technol. A 21, 1849 (2003).

${ }^{12}$ C. S. Corr, P. G. Steen, and W. G. Graham, Plasma Sources Sci. Technol. 12, 265 (2003).

${ }^{13}$ C. S. Corr, P. G. Steen, and W. G. Graham, Appl. Phys. Lett. 86, 141503 (2005).

${ }^{14}$ R. Kawai and T. Mieno, Jpn. J. Appl. Phys., Part 2 36, L1123 (1997).

${ }^{15}$ P. Chabert, T. E. Sheridan, R. W. Boswell, and J. Perrin, Plasma Sources Sci. Technol. 8, 561 (1999).

${ }^{16}$ R. N. Franklin and J. Snell, J. Phys. D 32, 1031 (1999).

${ }^{17}$ M. A. Lieberman and A. J. Lichtenberg, Principles of Plasma Discharges and Materials Processing (Wiley, New York, 1994).

${ }^{18}$ S. Samukawa, Appl. Phys. Lett. 64, 3398 (1994).

${ }^{19}$ R. W. Boswell, Phys. Lett. 33A, 470 (1970).

${ }^{20}$ A. Cantin and R. R. R. Gagne, Appl. Phys. Lett. 30, 31 (1977).

${ }^{21}$ M. B. Hopkins and W. G. Graham, Rev. Sci. Instrum. 57, 2210 (1986).

${ }^{22}$ T. E. Sheridan, P. Chabert, and R. W. Boswell, Plasma Sources Sci. Technol. 8, 457 (1999).

${ }^{23}$ M. Tuszewski and R. White, Plasma Sources Sci. Technol. 11, 338 (2002).

${ }^{24}$ M. Shindo, S. Uchino, R. Ichiki, S. Yoshimura, and Y. Kawai, Rev. Sci. Instrum. 72, 2288 (2001).

${ }^{25}$ L. St-Onge, M. Chaker, and J. Margot, J. Vac. Sci. Technol. A 18, 2363 (2000).

${ }^{26}$ S. Raul, P. L. G. Ventzek, I. C. Abraham, G. A. Hebner, and J. R. Woodworth, J. Appl. Phys. 92, 6998 (2002). 